\title{
Role of Technology \& Importance in Tracking Healthcare Services
}

\author{
G.R.Vaisshalli ${ }^{1} \&$ Apar Gupta ${ }^{2 *}$ \\ ${ }^{1,2}$ Blu Ocean Studios Private Limited, Uttar Pradesh-201301, India. \\ DOI: http://doi.org/10.38177/ajast.2022.6104
}

Copyright: () 2022 Apar Gupta et al. This is an open access article distributed under the terms of the Creative Commons Attribution License, which permits unrestricted use, distribution, and reproduction in any medium, provided the original author and source are credited.

The most fundamental expectation from the healthcare sector is that it provides a safe and reliable environment to serve patients. Medical supplies and equipment have also improved with technological advancements, making them easier to use, providing a better experience, and increasing their longevity. With advancement in technology, medical services can also be tracked for efficiency.

Keywords: Blu Ocean Innovations Private Limited, Healthcare Sector, Tracking, Technology.

\section{Introduction}

Patient tracking is a modern hospital technology that greatly simplifies control over patient flow, treatment progress, and basic hospital activities like patient acceptance and discharge. It increases the rate of bed turnover at the hospital, allowing doctors to treat more patients. This type of approach offers structure to patient visits and allows parents of minors peace of mind that their children are safe.

Improved healthcare outcomes, patient engagement, medication adherence, and other advantages of using information technology in hospitals are just a few of the advantages. Digital records, telemedicine, and other innovations push the boundaries, automate monotonous activities, and allow doctors and patients to communicate freely and easily.

The technology provides a plethora of possibilities for medical service tracking.

\section{Literature Review}

The technical study was in-depth and included montages of relationships and restrictions. IoT is one of the most important factor for healthcare services tracking. The basic purpose of the "Internet of Things" is to ensure that Internet-based communications and the sending and receiving of information are normally available when used in conjunction with "electronic sensor" devices. According to an estimate, by 2020, there will be 50.1 billion IoT users, up from 28.4 billion in 2017. According to scientific charity, "IoT" offers a variety of services. "Continuity of contact" is defined as "WiFi, cell phone, NFC, GPS, and so on." The main goal of the Internet of Things, however, is to incorporate organisations and mechanisation so that messages can be transmitted without interruptions, as opposed to software creation; the most frequently recycled sensors with accelerometers, compression-embedding camps such as the "MCUS, MPUs," are the start of the programmed. Amna Abdullah and colleagues exhibited a LabVIEW-based patient checking framework in their work project. The framework project was completed in five steps. To put the structure into action, they considered two frameworks. They connected the sensors attached to the patient's body to a transmitter unit connected to a Zig Beeor GSM orchestrate in the rule system. The information was transmitted remotely by the transmitter to a recipient who was also connected to a ZigBee or GSM engineer. 
The recipient was immediately connected to a USB port on a nearby monitoring unit (which is a Laptop with Lab VIEW programming in it).

\section{Technologies Used for Smart Tracking of Medical Services}

\subsection{Technologies for Patient Care}

Wearable technologies make it possible to track human physical activities and behaviours, as well as physiological and biochemical markers, in real time. Wearables such as wristbands and badges are used in hospitals to track a patient's location. A wristband has a code that, when scanned, grants access to patient records to a physician. This useful aspect of a wearable tracking system eliminates the agony of switching records - the eCW event alone serves as a reminder of how dangerous a lack of patient record structure can be.

For paramedics, equipment like this utilized in hospitals is a lifesaver. The ability to scan the most critical information about a patient in a matter of seconds - blood type, allergies, and so on - enhances field medical quality.

\subsection{Cloud Computing}

The practice of installing remote servers accessed via the internet to store, manage, and process healthcare-related data is known as cloud computing in healthcare. In contrast, setting up an on-site data centre with servers or hosting the data on a personal computer are both options. Client hospitals can share access to patient records and assure compatibility with cloud computing companies. The technology establishes a link between hospitals, patients, doctors, and other healthcare providers. Finally, hospitals may use the cloud to store all of their data and get a big-picture view of their facility's performance and patients' treatment progress.

Moreover, patients do not even tend to question the prescribed medicines or treatment. When there is lack of transparency in medical treatment, then it is one of the obvious challenge in healthcare sector and probably one of the most serious unattended issue!

\subsection{Real-Time Location System (RTLS)}

A Real-Time Location System (RTLS) in healthcare is a system that allows for the immediate or real-time tracking and management of medical equipment, employees, and patients in a variety of patient care settings.

\section{Importance of Tracking Medical Services}

\subsection{Improved Record Maintenance}

Real-time tracking provides clinicians with timely vitals and lab results, as well as insights into how to increase clinical trial efficiency and make a patient's hospital stay more productive. Aside from that, patient tracking guarantees interoperability among hospital units like physicians, surgeons, nurses, other healthcare professionals.

\subsection{Better Patient Queuing}

Hospital management will be able to establish whether there are any patterns in patient flow growth by tracking patients. Furthermore, tracking systems will alert a caregiver when a patient arrives for an appointment, reducing the need for receptionists and reducing the margin for error. 


\subsection{Improving Patient Satisfaction}

Tracking systems are an excellent illustration of how technology may help improve patient care. Healthcare facilities typically have a high patient turnover rate, with thousands of people passing through each month. Unfortunately, the majority of these consultations are one-time only; patients frequently miss follow-up sessions and develop chronic diseases as a result.

A hospital's objective should be to create a bond that helps patients become more self-aware. Tracking systems assist physicians in achieving this goal by providing hospitals with a database of patients who have follow-up appointments booked. A patient, on the other hand, will receive a phone call, an email, and a social media reminder on a future doctor's appointment.

\section{Conclusion}

Patient tracking enhances patient safety, lowers hospital operational expenses, and allows patients to receive more effective care. Keeping track of thousands of individuals would be nearly impossible without current technology, which is where the Internet of Things comes in.

Hospital managers can monitor patients' vitals and positions in real time, communicate with caregivers on a deeper level, and ensure that the treatment system runs smoothly with the development of a patient tracking system.

\section{Declarations}

\section{Source of Funding}

This research did not receive any grant from funding agencies in the public, commercial, or not-for-profit sectors.

\section{Competing Interests Statement}

The authors declare no competing financial, professional and personal interests.

\section{Consent for publication}

Authors declare that they consented for the publication of this research work.

\section{References}

[1] B. Schwald, H. Seibert and T. Weller, "A flexible tracking concept applied to medical scenarios using an AR window," Proceedings. International Symposium on Mixed and Augmented Reality, 2002, pp. 261-262, DOI: 10.1109/ISMAR.2002.1115102.

[2] K. Kim, J. Jun, S. Kim and B. Y. Sung, "Medical Asset Tracking Application with Wireless Sensor Networks," 2008 Second International Conference on Sensor Technologies and Applications (sensorcomm 2008), 2008, pp. 531-536, DOI: 10.1109/SENSORCOMM.2008.87.

[3] C. Cheng et al., "Accurate Location Tracking Based on Active RFID for Health and Safety Monitoring," 2009 3rd International Conference on Bioinformatics and Biomedical Engineering, 2009, pp. 1-4, DOI: 10.1109/ICBBE. 2009.5162266. 
[4] T. Lam, H. Yuan and M. C. de Oliveira, "Low-Cost Open-Source Solution to Optimize Emergency Medical Services in Developing Communities by Tracking, Dispatching, and Simulating," 2019 IEEE Global Humanitarian Technology Conference (GHTC), 2019, pp. 1-8, DOI: 10.1109/GHTC46095.2019.9033058.

[5] G. Wang, F. Ye, N. Zhang and M. Wang, "Design and implementation of medical service robot with single arm and tracking function," 2013 IEEE International Conference on Information and Automation (ICIA), 2013, pp. 840-843, DOI: 10.1109/ICInfA.2013.6720410. 